\title{
On the taxonomy of the genus Nyctycia Hampson, 1905 (Lepidoptera, Noctuidae)
}

\author{
BALÁZs BENEDEK ${ }^{1 *} \&$ BALÁZs TóTH ${ }^{2}$ \\ ${ }^{1}$ H-2045 Törökbálint, Árpád u. 53, Hungary \\ E-mail: benedekia@gmail.com; https://orcid.org/0000-0001-9533-1176 \\ ${ }^{2}$ Department of Zoology, Hungarian Natural History Museum, \\ Baross utca 13, H-1088, Budapest, Hungary \\ E-mail: toth.balazs@nhmus.hu; https://orcid.org/0000-0002-6089-1218
}

\begin{abstract}
The updated check-list of the genus Nyctycia Hampson, 1905 is presented. The male genitalia of Nyctycia lama Hreblay \& Ronkay, 1999, Nyctycia niveifera Hreblay \& Ronkay, 1999 and Nyctycia nigriclava Hreblay \& Ronkay, 1999 are described and illustrated. New distributional data for Vietnam is published for Nyctycia niveifera. The female genitalia of the subspecies Nyctycia strigidisca vargai Ronkay, Ronkay, Gyulai \& Hacker, 2010 are described and illustrated and the taxonomical problems of this taxon are discussed based on new distributional data and genitalia examinations. With 23 figures.
\end{abstract}

Key words - China, distributional data, Himalaya, Nepal, Noctuidae, Nyctycia, Xyleninae, taxonomy, Vietnam

\section{INTRODUCTION}

The genus Nyctycia has been erected by Hampson (1906) for the species Bombycia persimilis Hampson, 1894 and N. plagiogramma Hampson, 1906. This latter species has been transferred to a new genus, Nyctyciomorpha by Hacker \& Ronkay (1996). Moore (1881) described the species Apamea strigidisca Moore, 1881 which was transferred to the new genus Pachypolia Hampson, 1906 under the name P. himalayensis by HAMPSON (1906). In 1913, WARREN erected the genus Isopolia Warren, 1913 for Pachypolia himalayensis Hampson, 1906 (WARREN 1913). Finally, Isopolia has been synonymized by RoN KAY (1990) with Nyctycia. Up to date Nyctycia is consisting 31 species divided into six species-groups (HACKER \& RONKAY 1996, HREBLAY \& RONKAY 1999).

* corresponding author. 
Nyctycia is one of the characteristic late-autumn - winter flying genera of the greater Himalayan region. Species of Nyctycia occur in Afghanistan (1), Pakistan (2), India (data deficient), Nepal (17), Bhutan (data deficient), Myanmar (data deficient), Vietnam (6), Thailand (5), China (11), Taiwan (6), South Korea (1) and Japan (3). The characterization and taxonomy of the genus have been discussed by BOURSIN (1958), SUGI (1959), OWADA (1983) and HACKER \& RONKAY (1996).

Present paper is aimed to complement these taxonomical studies by the description of the genitalia of the previously unknown males of Nyctycia lama Hreblay \& Ronkay, 1999, Nyctycia niveifera Hreblay \& Ronkay, 1999 and Nyctycia nigriclava Hreblay \& Ronkay, 1999. The taxonomic problems of the subspecies Nyctycia strigidisca vargai Ronkay, Ronkay, Gyulai \& Hacker, 2010 are discussed.

\section{MATERIALS AND METHODS}

Specimens were collected using artificial light and were examined using modern dissection standards for Lepidoptera genitalic preparation. Genitalia were stained with eosin. Adults were photographed with an Olympus Camedia 7070 digital camera, genitalia slides with an Olympus DP70 photographic microscope, using the softwares DPController and DPManager. Images were adjusted and plates were prepared with the software Adobe Photoshop CS6.

Species group divisions of Nyctycia follow the publications of HACKER \& RonkAy (1996) and Hreblay \& Ronkay (1999), with the aim to place taxa presumably closely related in the same categories.

Abbreviations used: $\mathrm{BBT}=$ private collection of Balázs Benedek (Törökbálint, Hungary); HNHM = collection of the Hungarian Natural History Museum (Budapest, Hungary); GRB = private collection of Gábor Ronkay, (Budapest, Hungary); $\mathrm{MH} / \mathrm{HNHM}=$ collection of Márton Hreblay in the Hungarian Natural History Museum, (Budapest, Hungary); JB = genitalia preparation by János Babics; $\mathrm{MH}=$ genitalia preparation by Márton Hreblay; $\mathrm{RL}=$ genitalia preparation by László Ronkay; $\mathrm{TB}=$ genitalia preparation by Balázs Tóth. 


\section{RESULTS}

Ordo LEPIDOPTERA Linnaeus, 1758 Superfamily NOCTUOIDEA Latreille, 1809

Family NOCTUIDAE Latreille, 1809

Subfamily XYLENINAE Guenée, 1852

Tribus XYLENINI Guenée, 1837

Genus Nyctycia Hampson, 1906

Type species: Bombycia persimilis Hampson, 1894

\section{Check-list of Nyctycia}

Nyctycia persimilis species-group

- N. persimilis (Hampson, 1894)

- N. laci Ronkay, 1990

- N. decorata Hacker \& Ronkay, 1996

- N. lama Hreblay \& Ronkay, 1999

- N. nigriclava Hreblay \& Ronkay, 1999

- $\quad$. vernalis Hreblay \& Ronkay, 1998

- N. flavipicta (Hampson, 1906) ssp. zita Hreblay \& Ronkay, 1999

- N. mesomelana (Hampson, 1906) ssp. formosana Kobayashi \& Hreblay, 1998

- $\quad$. sugii Ronkay, 1990

Nyctycia strigidisca species-group

- $\quad$ N. strigidisca (Moore, 1881) ssp. owadai (Yoshimoto, 1988) ssp. nigridorsi Kobayashi, 1998 ssp. vargai Ronkay, Ronkay, Gyulai \& Hacker, 2010

- N. stenoptera (Sugi, 1959) ssp. minori Kobayashi, 1998 ssp. violascens Hreblay, Peregovits \& Ronkay, 1999 ssp. pygmaea Hreblay \& Ronkay, 1999

- $\quad$. parvula Kobayashi \& Owada, 1998

- $\quad$ N. angustipennis Yoshimoto, 1993

- N. szabokyi Hreblay, Peregovits \& Ronkay, 1999

Nyctycia latibasalis species-group

- N. latibasalis (Warren, 1913)

- $\quad$. muscipennis Owada, 1983 
- N. endoi (Owada, 1983)

ssp. hiemalis Hreblay, Peregovits \& Ronkay, 1999

- N. shelpa Yoshimoto, 1994

- $\quad$. adnivis Kobayashi \& Owada, 1998

- $\quad$. nivescens Hreblay, Peregovits \& Ronkay, 1999

- N. hoenei (Boursin, 1958)

ssp. simonyi Hreblay, 1998

- $\quad$ N. karma Hreblay \& Ronkay, 1999

- $\quad$. ionochlora Hreblay, Peregovits \& Ronkay, 1999

Nyctycia plumbeomarginata species-group

- N. plumbeomarginata (Hampson, 1895)

- N. albivariegata Hreblay, Peregovits \& Ronkay, 1999

- $\quad$. niveifera Hreblay, Peregovits \& Ronkay, 1999

Nyctycia consimilis species-group

- $\quad$ N. consimilis Hreblay \& Ronkay, 1998

- $\quad$. asymmetrica Hreblay \& Ronkay, 1998

- $\quad$ N. dissimilis Hreblay, Peregovits \& Ronkay, 1999

- N. attila Hreblay \& Ronkay, 1999

Nyctycia pectinata species-group

- N. pectinata Draudt, 1950

Taxonomy

Nyctycia lama Hreblay \& Ronkay, 1999

(Figs. 1-3, 13, 18)

Nyctycia lama - Hreblay \& Ronkay (1999): 534, holotype female, slide No. MH9884f (MH/HNHM); type-locality: West Nepal, $14 \mathrm{~km} \mathrm{~N}$ of Dailekh, $2600 \mathrm{~m}$.

Nyctycia lama Hreblay \& Ronkay - BÁlint, Katona \& GubÁnYi 2014: 191, fig. 228.

Material examined ( $\mathrm{n}=4$ ) - holotype female (Fig. 3); 1 male, 1 female, West-Nepal, Bheri, Dailekh area, $11 \mathrm{~km} \mathrm{~N}$ of Dailekh, $2380 \mathrm{~m}, \mathrm{~N} 28^{\circ} 56.322^{\prime}$, E8143.750', 21-22. XI. 2019 (Fig. 1), slide Nos. TB2024f (Fig. 18), TB2025m (Fig. 13); 1 female, West-Nepal, Bheri, Dailekh area, $13 \mathrm{~km} \mathrm{~N}$ of Dailekh, 2425 m, N2857.242‘, E8145.699`, 18. XI. 2019 (Fig. 2) (coll. BBT). 
Male genitalia - Uncus short and narrow, finger-like with subapical hairs present, tegumen high and narrow, vinculum relatively short but strong, U-shaped, fultura strong, lyriform, with long, densely setose arms, valva narrow and elongated, distally slightly dilated, sacculus short, harpe partly fused with very long and acute costal process, thorn-like, slightly curved, cucullus rounded, gently hairy. Aedeagus short, cylindrical, carina with a small ventral tooth, vesica everted straight, relatively short, sack-like with a large, dorsal side with rounded diverticulum, medial section of vesica covered with a dorsal side large but fine spinulose field, terminal section of vesica narrow, tubular (Fig. 13).

\section{Nyctycia nigriclava Hreblay \& Ronkay, 1999}

(Figs. 4-6, 14, 19)

Nyctycia nigriclava - HREBLAY \& RONKAY (1999): 535, holotype male, slide No. MH9872f (MH/HNHM); type-locality: West Nepal, $20 \mathrm{~km} \mathrm{~N}$ of Dailekh, $3000 \mathrm{~m}$.

Nyctycia nigriclava Hreblay \& Ronkay - BÁLInt, Katona \& GUBÁNYi 2014: 191, fig. 229.

Material examined $(\mathrm{n}=41)$ - holotype female (Fig. 5); 1 female, West-Nepal, Bheri, Dailekh area, $13 \mathrm{~km} \mathrm{~N}$ of Dailekh, $2425 \mathrm{~m}, \mathrm{~N} 28^{\circ} 57.242^{\prime}$, E81 ${ }^{\circ} 45.699^{\prime}$, 15. XI. 2019; 1 female, West-Nepal, Bheri, Dailekh area, $12 \mathrm{~km} \mathrm{~N}$ of Dailekh, 2650 m, N28 $57.4^{\circ}$, E8145.98‘, 16. XI. 2019; 1 female, West-Nepal, Bheri, Dailekh area, $20 \mathrm{~km} \mathrm{~N}$ of Dailekh, $3000 \mathrm{~m}, \mathrm{~N} 28^{\circ} 59.18^{\circ}$, E81 $44.50^{\circ}$, 17. XI. 2019; 3 males, 3 females, West-Nepal, Bheri, Dailekh area, $13 \mathrm{~km} \mathrm{~N}$ of Dailekh, 2425 m, N28 ${ }^{\circ} 57.242^{\prime}$, E81 ${ }^{\circ} 45.699^{\circ}, 18$. XI. 2019; 5 males, 6 females, WestNepal, Bheri, Dailekh area, $12 \mathrm{~km} \mathrm{~N}$ of Dailekh, $2650 \mathrm{~m}, \mathrm{~N} 28^{\circ} 57.4^{\prime}, \mathrm{E} 81^{\circ} 45.98^{‘}$, 19. XI. 2019, slide No. TB2027f (Fig. 19); 9 males, 10 females, West-Nepal, Bheri, Dailekh area, $11 \mathrm{~km} \mathrm{~N}$ of Dailekh, $2380 \mathrm{~m}, \mathrm{~N} 28^{\circ} 56.322^{\prime}$, E81 $43.750^{\circ}$, 21-22. XI. 2019 (Figs 4, 6), slide Nos. TB2026m, TB2028m, TB2029m (Fig. 14); 1 female, Nepal, Janakpur, Dolakha area, Thulopatal district, Salle village, 2250$2400 \mathrm{~m}, \mathrm{~N} 27^{\circ} 35.31^{\prime}, \mathrm{E} 86^{\circ} 9.23^{\prime}, 24-28$. XI. 2019 (coll. BBT).

Male genitalia - Uncus short and narrow, finger-like, apically pointed, subapical hairs missing, tegumen low and narrow, vinculum strong, broad, U-shaped, fultura triangular with thumb-like apical extension, valva narrow, elongated, sacculus small, clavus rounded, slightly dentate, harpe reduced, costal process well-developed, terminally formed into a large and strong, claw- or thorn-like process, cucullus more or less triangular in shape with corona present. Aedeagus short, cylindrical with coecum rounded, broader, carina with a dorsal side narrow ridge of small cornuti, vesica everted straight, relatively short, but broadly ovoid, ventral side basal diverticulum with a single small cornutus, vesica first half terminal part covered with a dorsal side narrow line of fine spiculi, terminal part of vesica narrower, tubular (Fig. 14). 
1

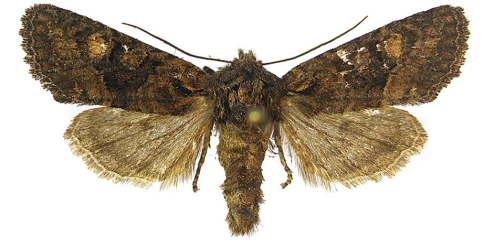

3

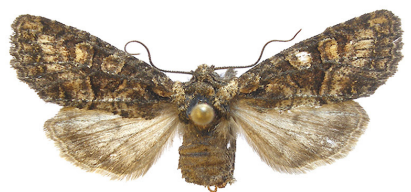

5
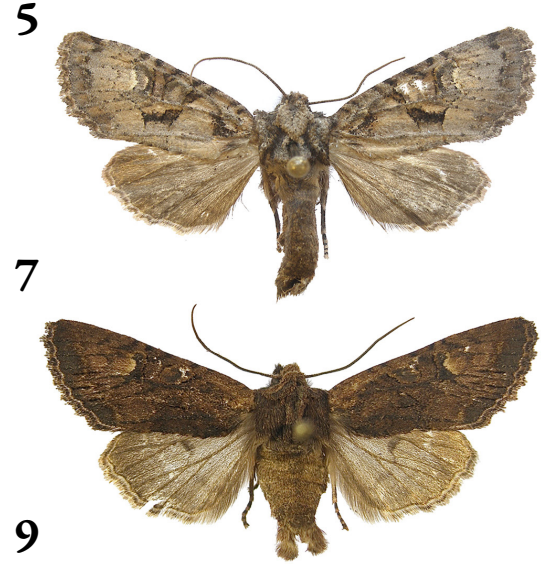

9

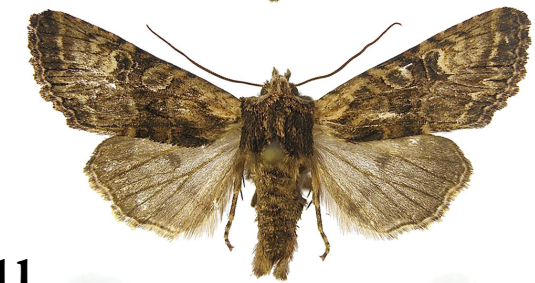

11

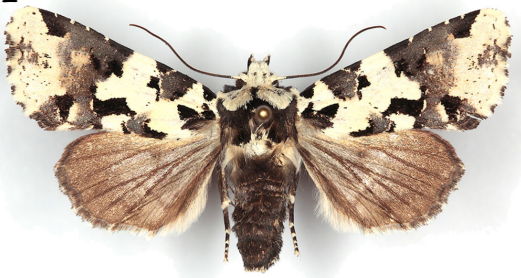

2

4
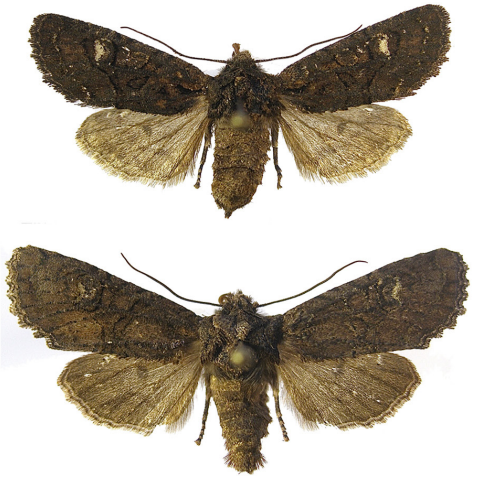

6
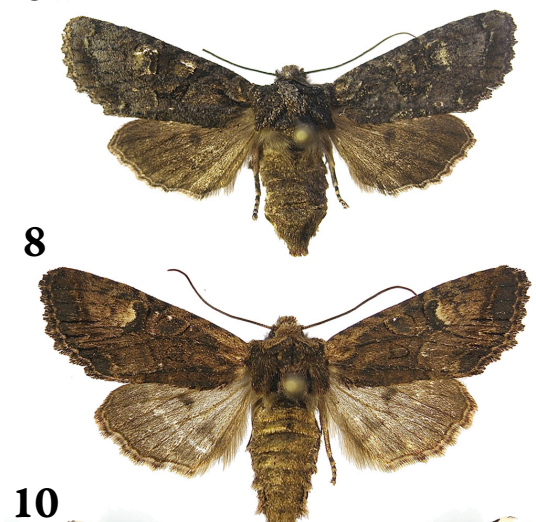

10

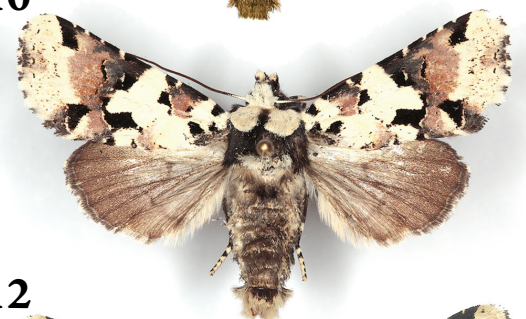

12

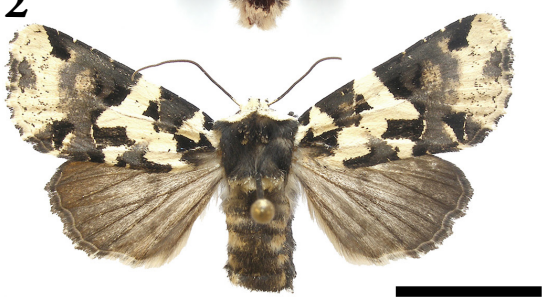

Figures 1-12. Adults of Nyctycia species. $1=N$. lama Hreblay \& Ronkay, 1999 male, West-Nepal; $\mathbf{2}=$ ditto, female, West-Nepal; $\mathbf{3}=$ ditto, holotype; $\mathbf{4}=$ N. nigriclava Hreblay \& Ronkay, 1999 male, West-Nepal; fig. $\mathbf{5}=$ ditto, holotype; $\mathbf{6}=$ ditto female, West-Nepal; $7=$ N. strigidisca vargai Ronkay, Ronkay, Gyulai \& Hacker, 2005 male, West-Nepal; 8 = ditto, female, West-Nepal; $9=N$. strigidisca strigidisca (Moore, 1881) male, West-Nepal; $10=N$. niveifera Hreblay \& Ronkay, 1999 male, Vietnam; $11=$ ditto, female, Vietnam; $12=$ ditto, holotype. Scale bar $=10 \mathrm{~mm}$. 


\section{Nyctycia strigidisca vargai Ronkay, Ronkay, Gyulai \& Hacker, 2010}

(Figs. 7, 8, 15, 20)

Nyctycia strigidisca vargai - RoNKAY et al. (2010): 271, holotype male, slide No. RL8777m (GRB); type-locality: Pakistan, Kashmir, Himalaya Mts., 30 km N Murree, Ayubia.

Material examined $(n=4)$ - paratype males, Pakistan, Kashmir, Himalaya Mts., $30 \mathrm{~km} \mathrm{~N}$ Murree, Ayubia; India, Himachal Pradesh, $15 \mathrm{~km} \mathrm{~W}$ of Shimla, slide Nos. RL9271m, RL9239m, (coll. HNHM); 1 male, 1 female, West-Nepal, Bheri, Dailekh area, $12 \mathrm{~km} \mathrm{~N}$ of Dailekh, $2650 \mathrm{~m}, \mathrm{~N} 28^{\circ} 57.4^{\prime}, \mathrm{E} 81^{\circ} 45.98^{\prime}, 19$. XI. 2019 (Figs 7, 8), slide Nos. TB2032m (Fig. 15), TB2033f (Fig. 20) (coll. BBT).

Female genitalia - Ovipositor small, conical, strongly sclerotized, densely and shortly setose, apophyses anteriores medium long, straight, apophyses posteriores short, ostium broad, funnel-shaped, antrum conical, with slightly curved edge, ductus bursae short and curved, left side strongly sclerotized, right edge ribbed, cervix bursae large, rounded and gently sclerotized, corpus bursae large, ovoid with two vertical and one horizontal, well developed signum bands (Fig. 20).

\section{Nyctycia niveifera Hreblay \& Ronkay, 1999}

(Figs. 10-12, 17, 22)

Nyctycia niveifera - HREBLAY \& RONKAY (1999): 23, holotype female, slide No. MH10750f, (MH/HNHM); type-locality: Thailand, Changwat Nan, $25 \mathrm{~km}$ of Bo Luang, $1150 \mathrm{~m}$.

Nyctycia niveifera Hreblay \& Ronkay - BÁLInt, Katona \& GUbÁnYi 2014: 191, fig. 230.

Material examined $(\mathrm{n}=3)$ - holotype female (Fig. 12); 1 male, Vietnam, Thua Thien Hue, Bach Ma Mt., 1400 m, March, 2016, leg, Thanh Luong Le (Fig. 10), slide No. JB2436m (Fig. 17); 1 female, Vietnam, Prov. Lam Dong, Lac Doung district, Bidoup Nui Ba National Park, 8. III. 2016, leg. Nhon Vo (Fig. 11), slide No. JB2440f (Fig. 22) (coll. BBT).

Male genitalia - Uncus short and small, apically pointed, subapical hairs present, fine, ridge-like, tegumen low, rounded, penicular lobes small, covered with very long and dense setae, vinculum strong, medium long but narrow, U-shaped, fultura inferior a long and broad bar, fultura superior oval in shape with raspy surface and with two, horn-like apical processes, sacculus large, more or less conical in shape, harpe small and short, thumb-like with long and strong, slightly curved basal bar, dorsal process characteristic, well developed, long and strong, medially turned and terminally rounded, valva elongated, cucullus triangular in shape, apically acute with corona present by long setae, subapical process well developed, thorn-like. Aedeagus long with coecum rounded and 
somewhat broader, carina a long but narrow bar on the ventral side, vesica everted ventrally, tubular, strongly twisted, covered with different kinds of setae and ribs on segment edges, medial diverticulum narrow, triangular, bears a thornlike terminal cornutus, medial section of vesica with an additional small, conical diverticulum with raspy surface and a large ridge of long, dense, brush-like setae (Fig. 17).

13

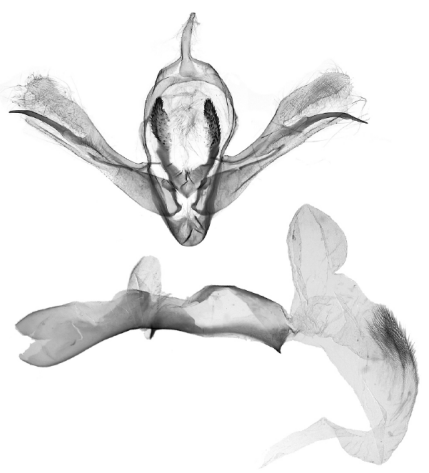

\section{5}

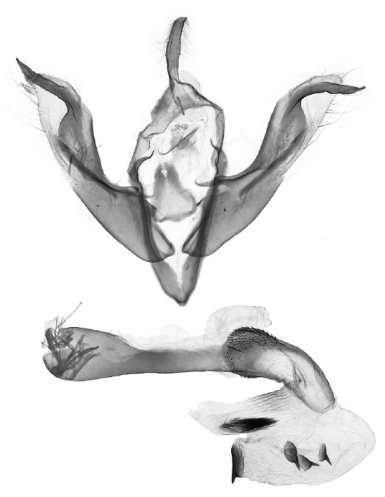

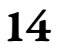
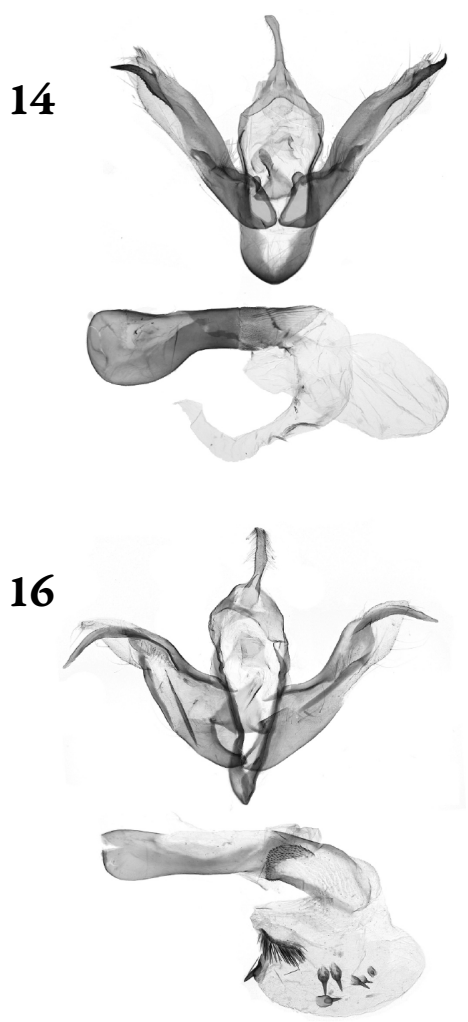

\section{7}

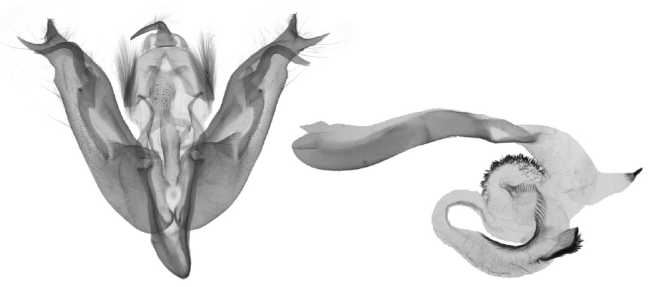

Figures 13-17. Male genitalia of Nyctycia species. $13=N$. lama Hreblay \& Ronkay, 1999, WestNepal, slide No. TB2025m; $14=$ N. nigriclava Hreblay \& Ronkay, 1999, West-Nepal, slide No. TB2029m; 15 = N. strigidisca vargai Ronkay, Ronkay, Gyulai \& Hacker, 2005, West-Nepal, slide No. TB2032m; 16 = N. strigidisca strigidisca (Moore, 1881), West-Nepal, slide No. TB2034m; $17=$ N. niveifera Hreblay \& Ronkay, 1999, Vietnam, slide No. JB2436m. Scale bar $=1 \mathrm{~mm}$. 


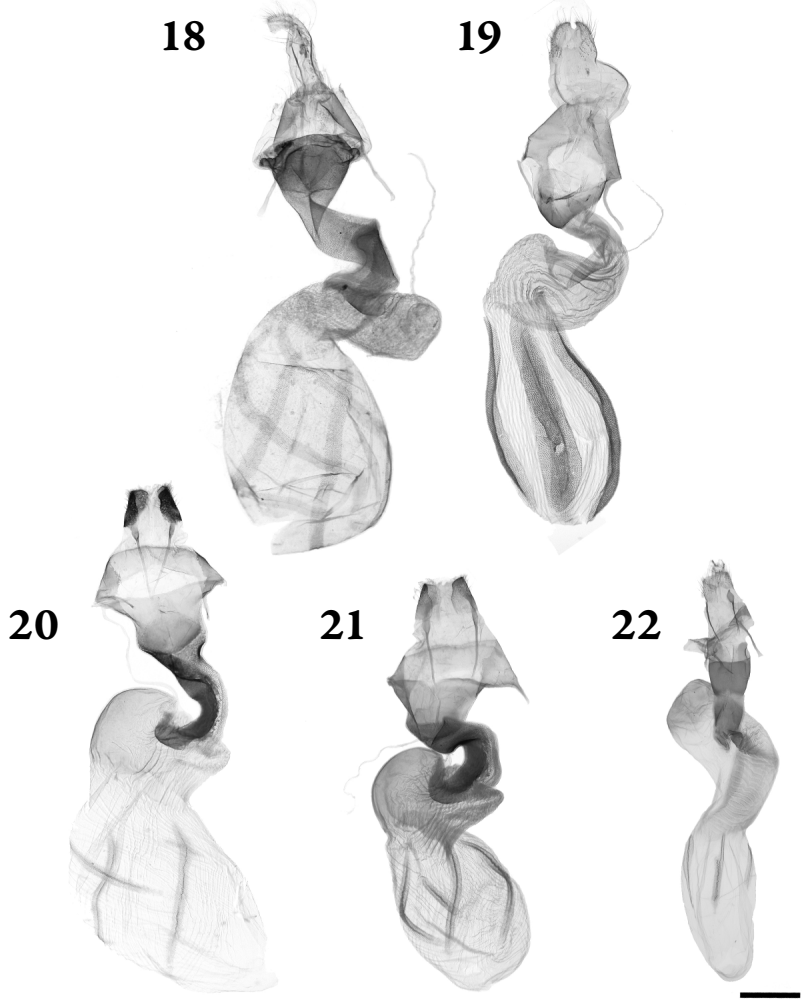

Figures 18-22. Female genitalia of Nyctycia species. $18=N$. lama Hreblay \& Ronkay, 1999, West-Nepal, slide No. TB2024f; $19=$ N. nigriclava Hreblay \& Ronkay, 1999, West-Nepal, slide No. TB2027f; 20 = N. strigidisca vargai Ronkay, Ronkay, Gyulai \& Hacker, 2005, West-Nepal, slide No. TB2033f; 21 = N. strigidisca strigidisca (Moore, 1881), West-Nepal, slide No. TB2046f;

$22=N$. niveifera Hreblay \& Ronkay, 1999, Vietnam, slide No. JB2440f. Scale bar $=1 \mathrm{~mm}$.

\section{DISCUSSION}

Associating opposite sexes - The co-occurrence of male and female specimens as well as their shared wing pattern elements enabled us to assign them to the same species. Sexual dimorphism is limited in the tribe, it is expressed only by slightly different shape of the wings (female wings usually shorter and broader) and body size (females tend for having larger abdomens) (see Figs. 1-12).

Collecting sites - Three Nyctycia taxa diagnosed above in details and documented in the Figures 1-22 were found north of Dailekh. One of the collecting sites is shown on Figure 23. The lamps were operated in forest clearings of the mountain ridge at elevations from 2380 to $3000 \mathrm{~m}$, between $15^{\text {th }}$ and $22^{\text {nd }}$ of November, 2019. The vegetation was mountainous Eastern Himalayan broadleaf forests dominated by Rhododendron and Quercus. 
Nyctycia strigidisca vargai - This subspecies has been described from Pakistan and the paratype series includes specimens from India (Himachal Pradesh). During the collecting trip in November, 2019, it has been found in West-Nepal (Dailekh area), together with nominotypical N. strigidisca specimens (Fig. 9). Our investigations of the male genitalia show differences only in the numbers and size of the cornuti on the vesica: the maximum number of cornuti on ssp. vargai is five (but in other two cases are only four), while on ssp. strigidisca there are seven or eight cornuti (Fig. 16). The female genitalia (Figs 20, 21) also show some slight differences in the configuration and sclerotization of the ductus bursae, but to have decisive evidence about the correct taxonomic rank of ssp. vargai, more material and DNA analysis would be necessary.

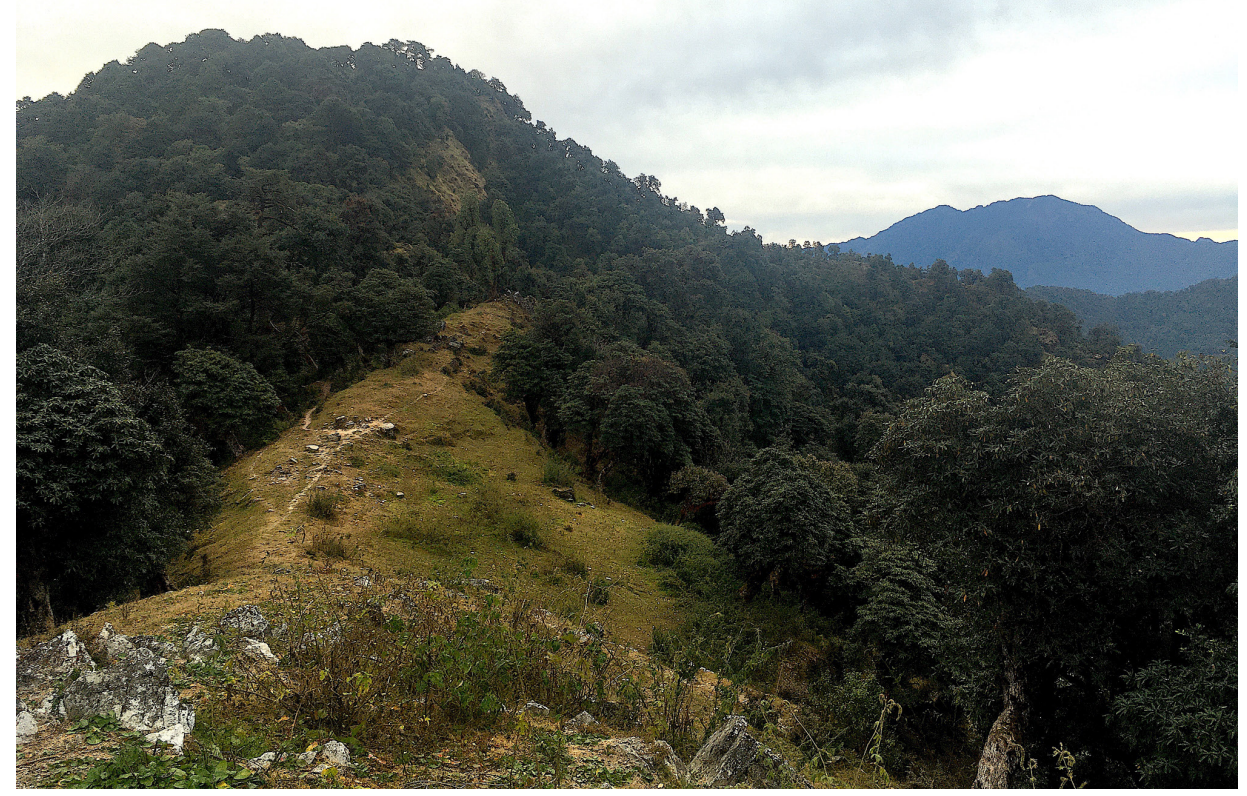

Figure 23. Collecting site of Nyctycia lama Hreblay \& Ronkay, 1999: West-Nepal, Bheri, Dailekh area, $13 \mathrm{~km} \mathrm{~N}$ of Dailekh, 2425 m, N2857.242‘, E8145.699', 15. XI. 2019.

(photograph by B. Benedek)

Acknowledgements - We are grateful to Mr János Babics (Páty) for the preparation of genitalia slides. 


\section{REFERENCES}

Bálint Zs., Katona G. \& Gubányi A. 2014: A life for Noctuidae, The owlet moths of Márton Hreblay (1963-2000). Names, specimens and types. - Hungarian Natural History Museum, Budapest, $270 \mathrm{pp}$.

Benedek B., Babics J. \& Saldaitis A. 2013: Taxonomic and faunistic news of the tribus Xylenini (s. 1.) (Lepidoptera, Noctuidae) from the greater Himalayan region. - Esperiana Buchreihe zur Entomologie 18: 7-38.

Boursin C. 1958: Zwei neue Cuculliinae aus Ostasien. Aus Dr. H. Höne's Ausbeuten. (Beiträge zur Kenntnis der „Noctuidae-Trifinae“, XCVI/96). - Zeitschrift der Wiener Entomologischen Gesellschaft 43: 45-48.

Draudt M. 1950: Beiträge zur Kenntnis der Agrotiden-fauna Chinas. - Mitteilungen der Münchner Entomologischen Gesellschaft 40(1): 1-174.

Hacker H. \& Ronkay L. 1992: Beschreibungen neuer Taxa der Spätherbst-Noctuidae-Fauna Zentralasiens und des Himalayaraumes (Cuculliinea sensu Hampson) (Lepiodoptera). Esperiana Buchreihe zur Entomologie 3: 193-221.

HACKer H. \& Ronkay L. 1996: Three new genera, seven new species and a general view on the late autumnal noctuid fauna of the Himachal Pradesh region of India (Lepidoptera, Noctuidae: Cuculliinae sensu Hampson). - Esperiana Buchreihe zur Entomologie 4: 337-360.

Hampson G. F. 1906: Catalogue of the Lepidoptera Phalaenae in the British Museum. Volume 6. Taylor and Francis, London, $532 \mathrm{pp}$.

Hampson G. F. 1908: Catalogue of the Lepidoptera Phalaenae in the British Museum. Volume 7. Taylor and Francis, London, 709 pp.

Hreblay M. \& Ronkay L. 1998: Noctuidae. In: Yoshimoto H. (ed.): Moths of Nepal, Part V. Tinea 15 (Supplement 1): 117-310.

Hreblay M. \& Ronkay L. 1999: Neue trifide Noctuidae aus dem himalayanischen Raum und der südostasiatischen Region (Lepidoptera: Noctuidae) - Esperiana Buchreihe zur Entomologie 7: 485-620.

Kobayashi H., Owada M. \& Hreblay M. 1998: On the genus Nyctycia Hampson, 1906 (Lepidoptera, Noctuidae, Cuculliinae) in Taiwan, with descriptions of two new species and three new subspecies. - Tinea 15(3): 256-265.

Moore F. 1882: Descriptions of new genera and species of Asiatic Lepidoptera Heterocera. Proceedings of the Scientific Meetings of the Zoological Society of London 1881: 326-380.

OwadA M. 1983: On the Cuculliinae genus Isopolia (Lepidoptera, Noctuidae) with descriptions of four new species. - Bulletin of the National Science Museum of Tokyo, A (Zoology) 9(1): 29-43.

Ronkay G., Ronkay L., Gyulai P. \& Hacker H. 2010: New Xylenini (Lepidoptera, Noctuidae, Xyleninae) species and genera from the wide sense Himalayan region. - Esperiana Buchreihe zur Entomologie 15: 245-358.

Ronkay L. 1990: Two new Nytycia Hampson, 1906 (= Isopolia Warren, 1913) (Lepidoptera, Noctuidae, Cucullinae) species from Korea and N India. - Esperiana Buchreihe zur Entomologie 1: 213-217. 
Shashank P. R. \& BenedeK B. 2020: New records of Noctuid moths (Lepidoptera, Noctuidae) from India, Bhutan and China. - Journal of Insect Biodiversity 20(1): 26-34.

Warren W. 1913: 2. Familie: Noctuidae. In: Seitz A. (ed.): Die Gross-Schmetterlinge der Erde. Eine systematische Bearbeitung der bis jetzt bekannten Gross-Schmetterlinge. Vol. 11, No. 14. - Alfred Kernen, Stuttgart, pp. 105-112.

\title{
A Nyctycia Hampson, 1905 génusz taxonómiájáról (Lepidoptera, Noctuidae)
}

\author{
BENEDEK BALÁZS ${ }^{1 *} \&$ Tóth BALÁZs ${ }^{2}$ \\ ${ }^{1} 2045$ Törökbálint, Árpád u. 53., Magyarország \\ E-mail:benedekia@gmail.com; https://orcid.org/0000-0001-9533-1176 \\ ${ }^{2}$ Magyar Természettudományi Múzeum Állattára, 1088 Budapest, Baross utca 13., Magyarosrszág \\ E-mail:toth.balazs@nhmus.hu;_https://orcid.org/0000-0002-6089-1218
}

Összefoglalás - A Nyctycia Hampson, 1905 génusz frissített fajlistáját közlik a szerzők. A Nyctycia lama Hreblay \& Ronkay, 1999, Nyctycia niveifera Hreblay \& Ronkay, 1999 és Nyctycia nigriclava Hreblay \& Ronkay, 1999 taxonok hím ivarszerve kerül leírásra és bemutatásra. A Nyctycia niveifera új elterjedési adatait jelzik Vietnámból. A Nyctycia strigidisca vargai Ronkay, Ronkay, Gyulai \& Hacker, 2010 alfaj nőstény ivarszervét leírják és bemutatják, továbbá besorolási problémáit vitatják meg az új elterjedési adatok és ivarszervi vizsgálatok eredményeinek fényében. 23 ábrával.

Kulcsszavak - elterjedési adatok, Himalája, Kína, Nepál, Noctuidae, Nyctycia, Xyleninae, taxonómia, Vietnám

\section{ÁBRAMAGYARÁZATOK}

1-12. ábrák. Nyctycia fajok imágói. $1=N$. lama Hreblay \& Ronkay, 1999 hím, Nyugat-Nepál; $\mathbf{2}=$ ditto nőstény, Nyugat-Nepál; $\mathbf{3}=$ ditto, holotípus; $\mathbf{4}=N$. nigriclava Hreblay \& Ronkay, 1999 hím, Nyugat-Nepál; fig. $\mathbf{5}=$ ditto, holotípus; $\mathbf{6}=$ ditto nőstény, Nyugat-Nepál; $7=N$. strigidisca vargai Ronkay, Ronkay, Gyulai \& Hacker, 2010 hím, Nyugat-Nepál; 8 = ditto nőstény, Nyugat-Nepál; $9=$ N. strigidisca strigidisca (Moore, 1881) hím, Nyugat-Nepál; $10=$ N. niveifera Hreblay \& Ronkay, 1999 hím, Vietnam; 11 = ditto nőstény, Vietnam; 12 = ditto, holotípus. Méretléc $=10 \mathrm{~mm}$.

* levelező szerző. 
13-17. ábrák. Nyctycia fajok hím ivarszervei. $13=N$. lama Hreblay \& Ronkay, 1999, NyugatNepál, gen. prep. TB2025m; 14 = N. nigriclava Hreblay \& Ronkay, 1999, Nyugat-Nepál, gen. prep. TB2029m; 15 = N. strigidisca vargai Ronkay, Ronkay, Gyulai \& Hacker, 2010, Nyugat-Nepál, gen. prep. TB2032m; $16=N$. strigidisca strigidisca (Moore, 1881), Nyugat-Nepál, gen. prep. TB2034m; $17=N$. niveifera Hreblay \& Ronkay, 1999, Vietnam, gen. prep. JB2436m. Méretléc $=1 \mathrm{~mm}$.

18-22. ábrák. Nyctycia fajok nőstény ivarszervei. $18=N$. lama Hreblay \& Ronkay, 1999, Nyugat-Nepál, gen. prep. TB2024f; $19=$ N. nigriclava Hreblay \& Ronkay, 1999, Nyugat-Nepál, gen. prep. TB2027f; $20=N$. strigidisca vargai Ronkay, Ronkay, Gyulai \& Hacker, 2010, Nyugat-Nepál, gen. prep. TB2033f; $21=$ N. strigidisca strigidisca (Moore, 1881), Nyugat-Nepál, gen. prep. TB2046f; $22=N$. niveifera Hreblay \& Ronkay, 1999, Vietnam, gen. prep. JB2440f. Méretléc $=1 \mathrm{~mm}$.

23. ábra. A Nyctycia lama Hreblay \& Ronkay, 1999 gyűjtőhelye: Nyugat-Nepál, Bheri, Dailekh area, $13 \mathrm{~km} \mathrm{~N}$ of Dailekh, $2425 \mathrm{~m}, \mathrm{~N} 28^{\circ} 57.242^{`}, \mathrm{E} 81^{\circ} 45.699^{\circ}, 15$. XI. 2019. (Benedek B. felvétele) 